\title{
Fourier compression: A customization method for quantum control protocols
}

\author{
Martín Larocca, ${ }^{*}$ Esteban Calzetta $\odot$, and Diego Wisniacki $\odot$ \\ Departamento de Física J. J. Giambiagi and IFIBA, FCEyN, Universidad de Buenos Aires, 1428 Buenos Aires, Argentina
}

(Received 2 March 2020; accepted 25 August 2020; published 16 September 2020)

\begin{abstract}
Quantum optimal control (QOC) is the field devoted to the production of external control protocols that actively guide quantum dynamics. Solutions to QOC problems were shown to constitute continuous submanifolds of control space. A solution navigation method exploiting this property to achieve secondary features in the control protocols was proposed [Larocca, Calzetta, and Wisniacki, Phys. Rev. A 101, 023410 (2020)]. Here, we present a navigation-powered protocol postprocessing mechanism allowing naturally inflexible optimization algorithms to produce user-customized solutions, for example, in terms of a Fourier decomposition. We test the performance of the method in two inherently different models: the two-level Landau-Zener system and the quantum harmonic oscillator.
\end{abstract}

DOI: 10.1103/PhysRevA.102.033108

\section{INTRODUCTION}

The second quantum revolution is expected to deliver new technology harvesting fundamentally quantum properties like entanglement and superposition, with applications in simulation, computing, sensing, and communication [1-6]. At the heart of quantum technology is quantum optimal control (QOC) theory [7-10]. The goal of QOC is to coherently control quantum dynamics. This is achieved by actively controlling an interaction between the system and a field (e.g., the electromagnetic field). The temporal profile of the interaction, $\omega(t)$, known as the control field, is shaped such that a given objective functional encoding the desired dynamics, $I[\omega(t)]$, is minimized. A parametrization is placed on the control field and the optimal parameters are found by performing local optimization routines. Gradient-based methods [11,12] usually outperform their gradient-free relatives [13] but, unlike the latter, are bounded to piecewise constant (PWC) Ansätze for the control field. Because of this, users requiring smooth and/or bandwidth-limited controls usually have to either include distorting penalty terms in the objective [14] or resort to the slower gradient-free methods that allow for arbitrary parametrizations on the control fields [15].

A methodology for studying the complexity involved in the search for solutions to QOC problems was developed in the early 2000s [16]. Quantum control landscapes (QCLs) are defined by the level hypersurfaces of the objective functional. Supposing the control field is parametrized by a vector of variables $\vec{\omega}$, the Hessian matrix,

$$
[H(\vec{\omega})]_{i, j}=\frac{\partial^{2} I(\vec{\omega})}{\partial \omega_{i} \partial \omega_{j}},
$$

can provide fundamental topological information. It has been shown $[17,18]$ that, for orthogonal-state-transfer control problems in systems with finite-dimensional Hilbert space, the

\footnotetext{
*1arocca@df.uba.ar
}

Hessian at a solution has an extensive null space and at most $2 D-2$ nonzero eigenvalues (here, $D$ is the dimension of the Hilbert space). Global optima constitute continuous submanifolds, level sets of control space $[19,20]$. Although general results for infinite-dimensional systems are still missing, in a recent publication [21], a frequency-driven quantum harmonic oscillator $(\mathrm{QHO})$ was studied and it was proven that, when targeting frictionless evolution, solutions form level sets with at most two directions of decreasing fidelity.

The existence of continuous manifolds of solutions has very interesting practical consequences. For example, it allows for the achievement of secondary features in the control protocols [21]. The idea is the following. An initial solution to the control problem can be further optimized with respect to a new cost function, without losing its initial fidelity, if the projection of the gradient of this auxiliary cost into the main objective solution manifold is used in a second descent procedure. Motion with this projected gradient generates fidelity-preserving trajectories with ever growing secondary yield.

Originally, the navigations were used to either smooth PWC protocols (penalizing the difference between successive pulses) or compress them into few-pulse solutions [21]. Here, we extend the compression idea to arbitrary bases (we exemplify with a Fourier basis, but using other bases is straightforward). The postprocessing provides fewparameter user-customized protocols, constituting a simple way of imposing (among many other conceivable constraints) smoothness and bandwidth limitations to the PWC protocols offered by standard gradient optimization algorithms. We benchmark the performance of the compression in two paradigmatic (and inherently different) models: the LandauZener (LZ) model and the QHO model.

The paper is organized as follows. Section II presents the physical models that will be used throughout the paper and Sec. III reviews the solution navigation method. Section IV presents the main result: a compression procedure to customize control protocols. We include some concluding 
remarks in Sec. V. Details of the numerical methods used are provided in Appendices A-C.

\section{MODELS}

Consider the evolution of an isolated two-level quantum system, described by the LZ Hamiltonian:

$$
\hat{H}[\omega(t)]=\frac{\Delta}{2} \hat{\sigma}_{x}+\omega(t) \hat{\sigma}_{z}
$$

with $\sigma_{x} / \sigma_{z}$ the usual Pauli matrices, $\Delta$ the minimal energy gap, and $\omega(t)$ the control field. This model describes, for example, a spin- $\frac{1}{2}$ particle in a magnetic field with a fixed $x$ component and a time-dependent $z$ one. We adopt natural units, setting $\hbar=1$, such that energy is measured in units of frequency. Suppose the control is initially set to $\omega=-\infty$ and the spin is prepared on the ground state of Eq. (2), that is, $|\psi(t \rightarrow-\infty)\rangle=|0\rangle$. For a linear sweep of $\omega$ over time, $\omega_{v}(t)=v t$, an analytical formula for the asymptotic probability of finding the ground state of $\hat{H}(t \rightarrow+\infty)=|1\rangle$ (that is, flipping the spin), can be derived [22]. Since energy levels become nearly degenerate at $\omega=0$, in order to suppress the probability of populating the excited level, the level crossing has to be traversed slowly, with $v \ll v_{c} \propto \Delta^{2}$. That is, narrow crossings demand slower protocols. Recent work [23] has considered a finite-time version of this problem, finding that the minimum time required to traverse a crossing is given by $T_{\min }=\pi / \Delta$.

Let us introduce the infidelity for the spin-flip task:

$$
I_{\mathrm{LZ}}[\omega(t)]=1-\left|\left\langle 1\left|\hat{U}_{T}[\omega(t)]\right| 0\right\rangle\right|^{2} .
$$

This functional maps real-valued functions $\omega(t)$ to real numbers $I_{\mathrm{LZ}}[\omega(t)]$ measuring the departure from the target state. The final-time propagator, $\hat{U}_{T}[\omega(t)]$, is the solution to the Schrödinger equation,

$$
i \frac{d \hat{U}_{t}}{d t}=\hat{H}[\omega(t)] \hat{U}_{t},
$$

evaluated at time $t=T$. We use PWC control fields:

$$
\omega(t)= \begin{cases}\omega_{1} & \text { if } 0<t<\Delta t \\ \vdots & \\ \omega_{M} & \text { if }(M-1) \Delta t<t<T\end{cases}
$$

where $\Delta t=T / M, T$ being the duration of the protocol and $M$ being the number of constant pulses.

Solutions to $D$-dimensional pure-state-transfer control problems present at most $M_{\min }=2 D-2$ directions of decreasing fidelity [19]. In this case, $D=2$ so $M_{\min }=2$. If $M=$ 2 variables are used, multiple solutions exist but are disconnected policies in the QCL [24]. Instead, if we choose $M \geqslant 3$, continuous submanifolds of solutions arise. To better understand this situation, we consider the case $M=3$, initializing 4000 seeds of the form $\vec{\omega}=\left(\omega_{1}, \omega_{2}, \omega_{3}\right)$, with $\omega_{i}$ randomly chosen from a uniform distribution in $[-5,5]$. The seeds are optimized with the cost function in Eq. (3). In Fig. 1, we plot those optimized fields that are globally optimal (with infidelity below a certain threshold, $I<I_{\text {th }}=10^{-6}$ ). A hidden structure is revealed. Solutions gather in closed looplike formations, appearing to form continuous curves. In the following section we will show how to use the navigation method to build a

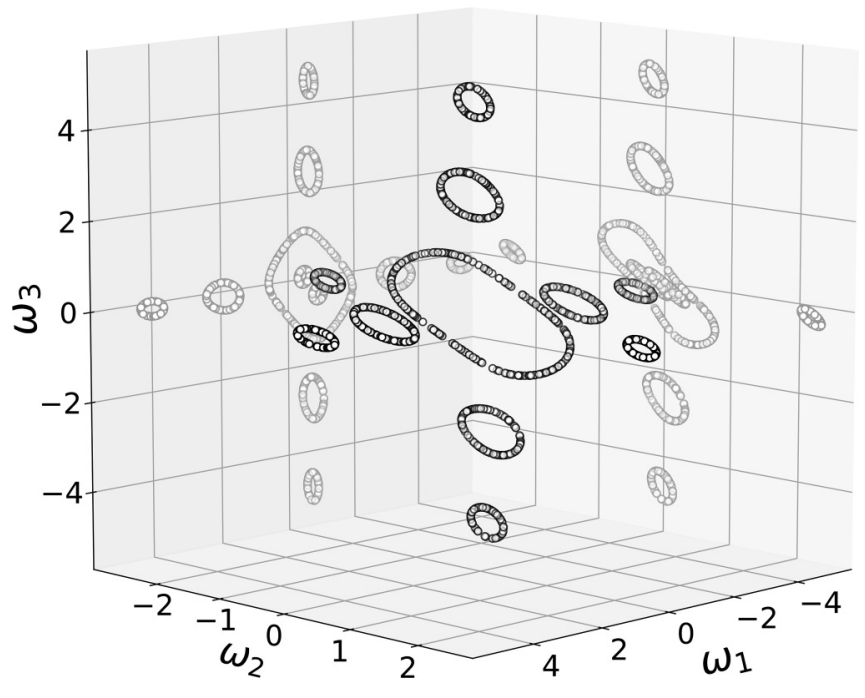

FIG. 1. Solution sets for the LZ model with $M=3$ control parameters. Each black point in $3 \mathrm{D}$ space represents a possible protocol, with $\omega_{i}$ the amplitude of the $i$ th pulse in the PWC sequence. Dim gray points represent the projections of the solutions onto the $Y Z$ and $X Z$ planes. We initialize thousands of random seeds and optimize with respect to the cost function of Eq. (3). Those optimized fields with infidelity below $10^{-6}$ are plotted as black dots. The solutions appear to form continuous curves, one-dimensional submanifolds of parameter space.

trajectory connecting these solutions. Figure 2 provides the spectrum of the Hessian at one of these optimal fields. Solutions are found to have exactly two nonzero eigenvalues. We further verify this showing the spectrum of a $M=48$ solution (inset). We have set $\Delta=1$ and $T=\frac{1.4 \pi}{2}$ in the simulations.

As a second model, regard a particle in a one-dimensional time-dependent harmonic trap, the evolution of which is described by the QHO Hamiltonian:

$$
\hat{H}(t)=\frac{\hat{p}^{2}}{2 m}+\frac{m}{2} \omega(t)^{2} \hat{x}^{2}
$$

where $\hat{x}$ and $\hat{p}$ are position and momentum operators, respectively; $m$ is the mass of the particle; $\omega(t)$ is the time-dependent frequency of the trap. Again, we set $m=\hbar=1$. Originally, the trap has frequency $\omega(0)=\omega_{0}$. In the context of quantum

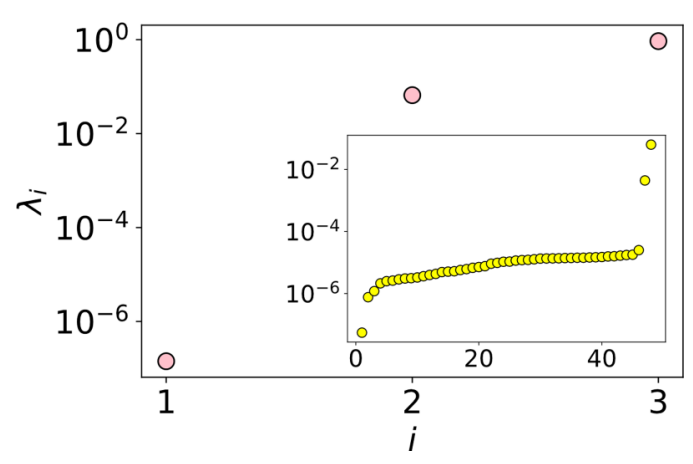

FIG. 2. Eigenvalues of the Hessian of Eq. (3) at a solution of $M=3$ ( $M=48$ in the inset). We observe exactly two nonzero eigenvalues, corresponding to those eigendirections that depart from the solution set. 
heat engines [25-29], a typical problem is to design adiabatic expansion or compression strokes, that is, finding protocols for opening or closing the trap, such that $\omega(T)=\omega_{T} \neq \omega_{0}$ and $N(T)=N(0), N$ being the particle number expectation value. In general, evolution with arbitrary driving protocols $\omega(t)$ causes the time-evolved Hamiltonian to no longer be diagonal in the basis of states with well-defined particle number, $\hat{N}(0)=\hat{a}^{\dagger}(0) \hat{a}(0)$. Nevertheless, a basis diagonalizing this time-evolved Hamiltonian can be obtained through a Bogoliubov transformation $[21,30]$ :

$$
\hat{a}(T)=\alpha \hat{a}(0)+\beta \hat{a}^{\dagger}(0)
$$

where $\alpha$ and $\beta$ are protocol-dependent complex coefficients satisfying $|\alpha|^{2}-|\beta|^{2}=1$. Initial states with a well-defined particle number (or incoherent superpositions of them) can only experience an increase in the mean particle number,

$$
N(T)=\left\langle\hat{a}^{\dagger}(T) \hat{a}(T)\right\rangle=N(0)\left(1+2|\beta|^{2}\right)+|\beta|^{2},
$$

a process called quantum friction in the literature [25-29]. A natural measure for the departure from target frictionless evolution is given by

$$
I_{\mathrm{QHо}}[\omega(t)]=|\beta|^{2}
$$

so we will use this as the objective functional. See Ref. [21] for a detailed description on how to compute the Bogoliubov coefficient $\beta$ associated with a given driving.

\section{SOLUTION NAVIGATION}

Reference [21] introduced a framework capable of navigating through solutions to control problems. Let us review the procedure. Consider a trajectory in parameter space, $\vec{\omega}(\zeta)$, the solution to an initial value problem:

$$
\frac{d \vec{\omega}(\zeta)}{d \zeta}=f[\vec{\omega}(\zeta)]
$$

with $\vec{\omega}(0)=\vec{\omega}_{0}$. Suppose $\vec{\omega}_{0}$ is a solution and let us choose $f[\vec{\omega}(\zeta)]$ to be $P \vec{a}$, with $\vec{a}$ an arbitrary vector and $P$ the projection onto the null subspace of $\leftrightarrow$, given by

$$
P \vec{a}=\vec{a}-\sum_{i \mid \lambda_{i} \neq 0} \vec{v}_{i} c_{i}
$$

The sum runs through the normalized Hessian eigenvectors $\vec{v}_{i}$ associated with nonzero eigenvalue $\lambda_{i}$, and $c_{i}=\vec{a} \cdot \vec{v}_{i}$. The arising trajectory is constrained to solution space. In practice, we use a fourth-order Runge-Kutta integration routine to numerically approximate Eq. (9) and a second-order finite-difference (FD) approximation to compute the Hessian matrix (see Appendix A for details). As an example, in Fig. 3 we use the method to drive through the solutions of Fig. 1. Starting from an optimal field, a perfect fidelity trajectory (red line) is built by following, at each iteration, the instantaneous eigenvector associated with the null Hessian eigenvalue (see Fig. 1).

\section{FOURIER COMPRESSION}

Previous work [21] demonstrated how to exploit the navigation procedure to smooth or compress solutions obtained from raw optimization. Smoothing procedures were shown to

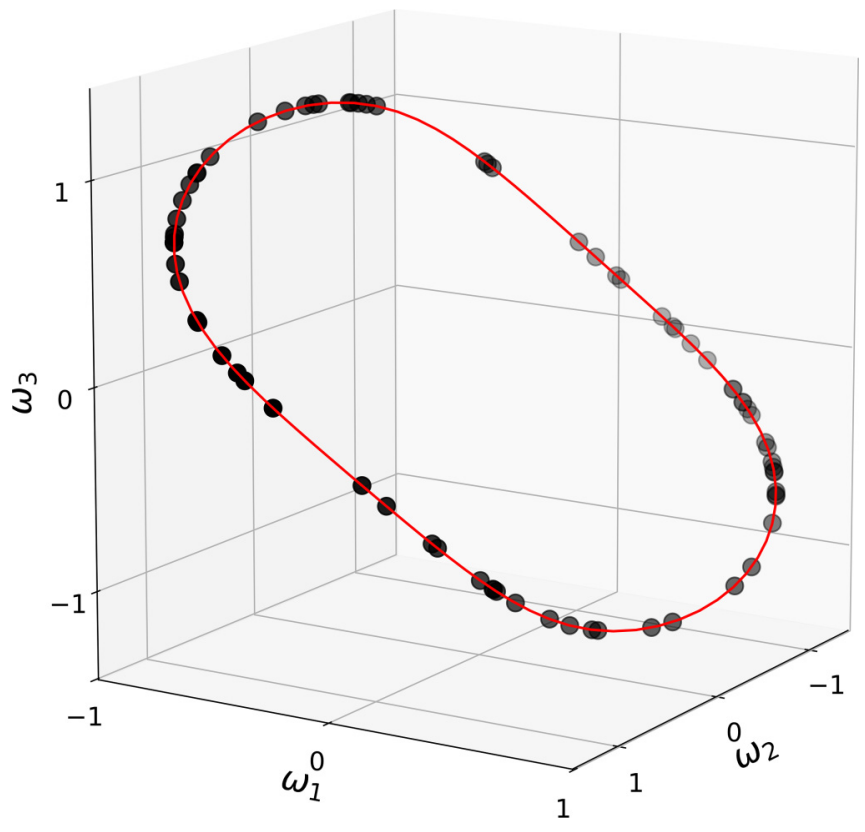

FIG. 3. Following the null Hessian eigenvector. Black dots depict solutions found optimizing thousands of initial random seeds (see Fig. 1). Starting from one of these solutions, we follow the FDgenerated Hessian eigenvector associated with the null eigenvalue generating a fidelity preserving trajectory (red curve) linking all of the scattered solutions. We set $\epsilon=10^{-2}$ and $h=0.01$.

be effective at producing regular control fields. Alternatively, protocols described by only a few parameters were obtained putting forward compression procedures. Since compression was performed in the original PWC parametrization, the two approaches were incompatible. Let us show how these two secondary features can be simultaneously tackled by extending the compression idea to the frequency realm.

First, let us introduce the general compression mechanism. The idea is the following. First, choose an orthonormal basis for control space $B=\left\{\phi^{j} \mid j \in[1, M]\right\}$. Since we are working with $M$-dimensional PWC control fields, the control space is $\mathbb{R}^{M}$, the $M$-dimensional vector space over the field of real numbers. Now, expand the initial field to be postprocessed in this basis:

$$
x=\sum_{j=1}^{M} a_{j} \phi^{j} .
$$

Suppose we want the field to have components only on $\bar{B}=\left\{\phi^{j} \mid j \in p\right\} \subset B$, for some subset of indices $p$. To enforce this compression, we define a cost penalizing the components outside $p$,

$$
C_{p}(x)=\sum_{k \notin p} y_{k}^{2}
$$

where $y_{k}$ is the component of $x$ on the $k_{\mathrm{th}}$ element of the basis,

$$
y_{k}=\left\langle x, \phi^{k}\right\rangle=\sum_{n=1}^{M} x_{n} \phi_{n}^{k},
$$

and follow its gradient, projected onto the solution submanifold [as explained in Eq. (10)]. 


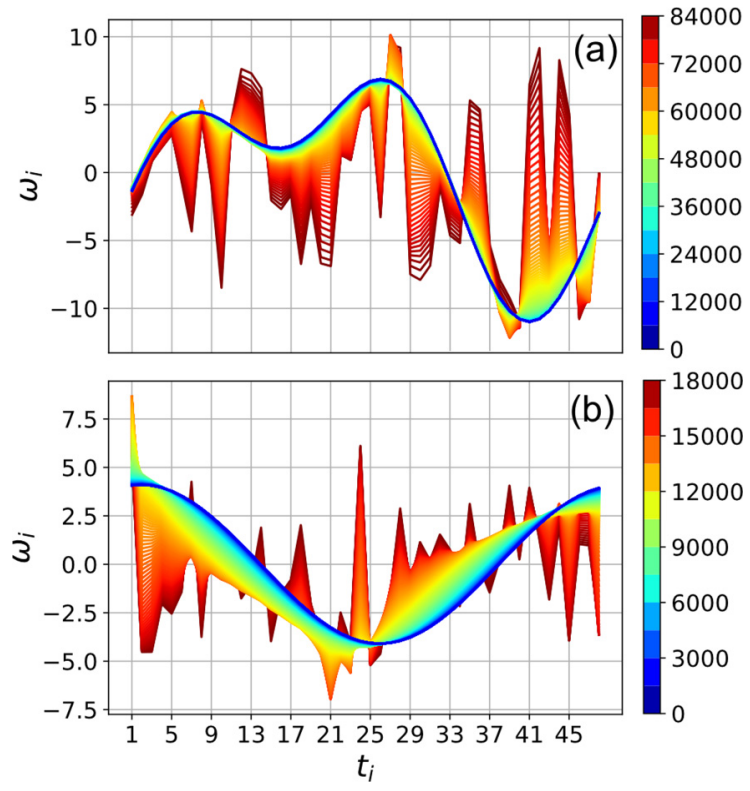

FIG. 4. Fourier protocol compression in the QHO. Each point in the graph depicts the value of the $i$ th component of a given protocol, corresponding to the time interval $\Delta t_{i}$. That is, the curves represent distinct protocols, which were colored relative to their secondary objective cost value of Eq. (12). All of them are optimal with respect to the main objective in Eq. (8). Starting with different random solutions, we present Fourier compression trajectories with (a) $p_{s}=p_{c}=\{1,2\}$ and (b) $p_{s}=p_{c}=\{1\}$.

In particular, for Fourier compression, we choose

$$
\begin{aligned}
B & =B_{c} \cup B_{s} \\
& =\left\{\cos \left(2 \pi k t_{n}\right) \mid k \in[0, M / 2]\right\} \\
& \cup\left\{\sin \left(2 \pi k t_{n}\right) \mid k \in[1, M / 2-1]\right\}
\end{aligned}
$$

where $t_{n}=n / M$. Let us use $p_{s}$ and $p_{c}$ to address the set of allowed indices for the sine and cosine bases individually. For example, $p_{s}=p_{c}=\{1\}$ indicates that we want the field to be a linear combination of sine and cosine with frequency 1. Figure 4 shows compression trajectories for the QHO control problem. We begin with $M=48$ solutions and follow the projected gradient of Eq. (12) for (a) $p_{s}=p_{c}=\{1,2\}$ and (b) $p_{s}=p_{c}=\{1\}$. Notice how the initial high-dimensional protocols are now described by only a few parameters (four and two, respectively). Let us refer to the latter case as full compression, since the final number of parameters describing the field equals $M_{\min }$, a lower bound on the number of parameters needed [20,31]. Further compression is very unlikely to succeed.

Similar compression trajectories for the LZ problem are presented in Fig. 5. Again, setting $p_{s}=p_{c}=\{1,2\}$ for trajectory (a) means we penalize every frequency in the control field except for the fundamental and first harmonic, while the $p_{s}=$ $p_{c}=\{1\}$ case in (b) reflects that we aim for a monochromatic field. In both situations, the Hessian was computed using a finite-difference second-order approximation. Details are provided in Appendix A. The simplicity of the models studied allows for the exact diagonalization of the PWC Hamiltonian,

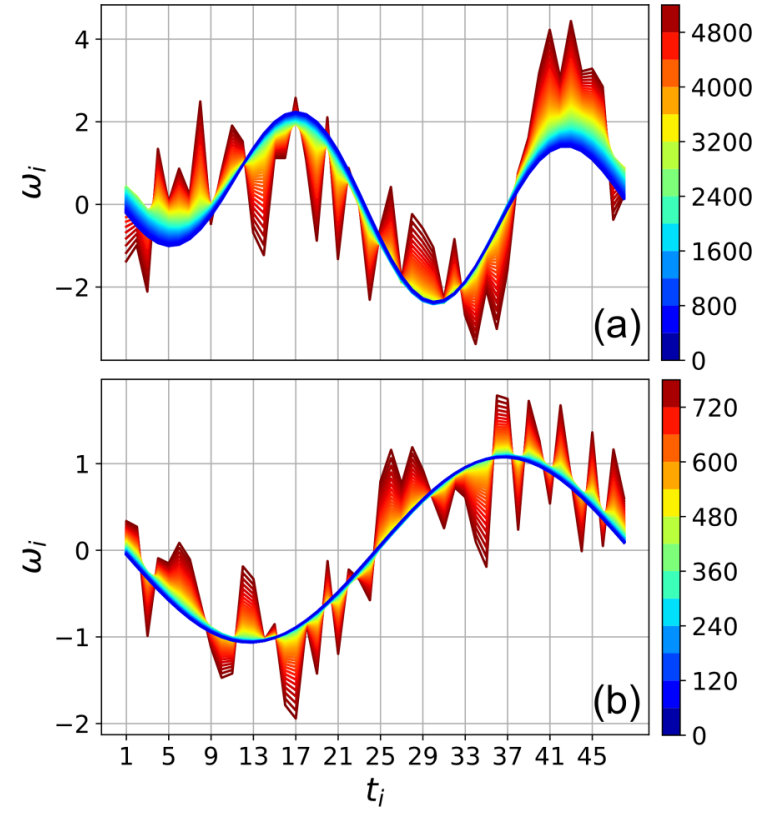

FIG. 5. Fourier protocol compression in the LZ model. Each point in the graph depicts the value of the $i$ th component of a given protocol, corresponding to the time interval $\Delta t_{i}$. The curves represent distinct protocols, which were colored relative to their secondary objective cost value of Eq. (12). All of them are optimal with respect to the main objective in Eq. (3). Starting with different random solutions, we present Fourier compression trajectories with (a) $p_{s}=p_{c}=\{1,2\}$ and (b) $p_{s}=p_{c}=\{1\}$.

and thus exact expressions for the propagator and its derivatives are available (see Appendix B). A benchmarking of the performance of the finite-difference approximations can be found in Appendix C.

\section{FINAL REMARKS}

Solutions to quantum control problems form continuously varying level sets in the QCL. Recent work evidenced that this property may have major practical consequences [21]. Navigation methods were shown to provide a straightforward way of producing secondary features in the control protocols (e.g., smoothness or compression).

Gradient-based QOC optimization methods lack the possibility of choosing the basis in which the control field is expanded. Albeit fast-converging, they produce protocols that are irregular due to the underlying fixed PWC parametrization. This is hard to reconcile with the natural bandwidth requirements of laboratory experiments. In this paper, we proposed a navigation-based protocol postprocessing procedure that allows the user to overcome this problem. Defining an auxiliary cost penalizing the Fourier components of the control protocol, initially irregular and high-dimensional solutions were evolved into smooth controls described by only a few parameters. Fourier compression constitutes an alternative approach to smoothness, bandwidth limitation, and protocol customization in quantum control.

Although originally developed to explore the complexity of the search for controls, the study of QCLs is proving to be more fruitful than expected. The results presented in this 
paper pave the way for the design of universal solution manifold navigation methods, extending their application beyond analytically solvable models.

\section{ACKNOWLEDGMENTS}

The authors acknowledge financial support from Agencia Nacional de Promoción de la Investigación, el Desarrollo Tecnológico y la Innovación (ANCyPT) (Grant No. PICT2016-1056), Consejo Nacional de Investigaciones Científicas y Técnicas (CONICET) (Grants No. PIP 11220170100817CO and No. PIP 11220150100493CO), and Secretaría de Ciencia y Técnica, Universidad de Buenos Aires (UBACyT) (Grants No. 20020130100406BA, 20020170100234BA and 20020170100129BA).

\section{APPENDIX A: APPROXIMATE HESSIAN COMPUTATION}

This Appendix provides the details regarding the FD computation of the Hessian matrix, used in Secs. III and IV to power the navigations. This is not the most efficient way of computing derivatives of the objective. We chose a model-free approach mainly because of its simplicity and all-terrain features. More efficient methods can be found in Refs. [32,33]. In particular, both models described in the main text are simple enough to allow for an exact treatment, which we describe in Appendix B. Finally, in Appendix C, we benchmark the quality of the approximations.

Consider the $\epsilon$-approximated central-difference Hessian matrix:

$$
\begin{aligned}
{\left[\mathrm{H}_{\epsilon}(\vec{\omega})\right]_{i, j}=} & \frac{1}{4 \epsilon^{2}}\left\{I\left[\vec{\omega}+\epsilon\left(\vec{e}_{i}+\vec{e}_{j}\right)\right]\right. \\
& -I\left[\vec{\omega}+\epsilon\left(\vec{e}_{i}-\vec{e}_{j}\right)\right] \\
& -I\left[\vec{\omega}-\epsilon\left(\vec{e}_{i}-\vec{e}_{j}\right)\right] \\
& \left.+I\left[\vec{\omega}-\epsilon\left(\vec{e}_{i}+\vec{e}_{i}\right)\right]\right\}+\delta_{\mathrm{FD}}
\end{aligned}
$$

where $\vec{e}_{i}$ is the $i$ th basis vector of parameter space and the error, $\delta_{\mathrm{FD}}$, is $O\left(\epsilon^{2}\right)$. A central-difference approach is about four times more expensive than a forward-difference one, but certainly worth it considering that the latter has an error that is $O(\epsilon)$. Of course in the limit $\epsilon \rightarrow 0$ both errors vanish. But this ignores the round-off errors, $\delta_{\mathrm{RO}}=O\left(u / \epsilon^{2}\right)$, that are introduced when the function $I$ is evaluated numerically [34]. Here, $u$ is an upper bound on the error arising in the casting of real numbers $x$ into floating-point numbers: $|x-f l(x)| \leqslant$ $x(1+u)$. We use double-precision floating-point arithmetic, where $u$, also known as unit round-off error or machine epsilon, is about $10^{-16}$. Because the round-off error is inversely proportional with $\epsilon$, a tradeoff takes place. An estimation of the optimal step, $\epsilon_{\mathrm{opt}}$, in terms of $u$ can be obtained minimizing the sum of the errors $\frac{d}{d \epsilon}\left[\delta_{\mathrm{RO}}(\epsilon, u)+\delta_{\mathrm{FD}}(\epsilon)\right]=0$. This gives $\epsilon \approx u^{1 / 4} \approx 10^{-4}$ and, in consequence, an upper bound to the error of $\approx 10^{-8}$.

\section{APPENDIX B: EXACT HESSIAN COMPUTATION}

In this section we obtain exact derivatives of the objective. We use an open-source symbolic computation library [35] to compute expressions for the propagator $U(\omega, t)$ as a function two symbolic variables $\omega$ and $t$. We start by building the
Hamiltonian matrix:

$$
H(\omega)=\sigma_{x}+\omega \sigma_{z}
$$

The simplicity of this system allows us to obtain exact expressions for the eigenenergies $\lambda_{k}(\omega)$ and eigenvectors $|k(\omega)\rangle$ of the Hamiltonian and, thus, via the spectral theorem, an exact expression for the propagator matrix:

$$
\begin{aligned}
U(\omega, t) & =e^{-i H(\omega) t} \\
& =\sum_{k} e^{-i \lambda_{k}(\omega) t}|k(\omega)\rangle\langle k(\omega)| .
\end{aligned}
$$

Finally, this expression is differentiated to obtain $\frac{d U(\omega, t)}{d \omega}$ and $\frac{d^{2} U(\omega, t)}{d \omega^{2}}$. The three symbolic expressions are converted to functions and saved to disk. Each time we want to compute the objective and its derivatives at a certain field $\vec{\omega}$, we load these building blocks and evaluate them. For example, the infidelity of a given $M$-dimensional PWC protocol

$$
I_{\mathrm{LZ}}=1-\left|\left\langle 1\left|U_{M} \ldots U_{1}\right| 0\right\rangle\right|^{2}
$$

involves $M$ constant-field propagators

$$
U_{k}=e^{-i\left(\sigma_{x}+\omega_{k} \sigma_{z}\right) \Delta t}
$$

that are easily obtained by evaluating the precomputed propagator with $(w, t)=\left(w_{k}, \Delta t\right)$. Similarly, the Hessian of the objective

$$
\begin{aligned}
\frac{\partial^{2} I_{\mathrm{LZ}}}{\partial \omega_{j} \partial \omega_{k}}= & -2 \operatorname{Re}\left[\left\langle 1\left|\frac{\partial^{2} U_{T}}{\partial \omega_{j} \partial \omega_{k}}\right| 0\right\rangle\left\langle 0\left|U_{T}^{\dagger}\right| 1\right\rangle\right. \\
& \left.+\left\langle 1\left|\frac{\partial U_{T}}{\partial \omega_{j}}\right| 0\right\rangle\left\langle 0\left|\frac{\partial U_{T}^{\dagger}}{\partial \omega_{k}}\right| 1\right\rangle\right]
\end{aligned}
$$

is ultimately written in terms of gradients and Hessians of constant-field propagators that are evaluated with the precomputed functions:

$$
\begin{gathered}
\frac{\partial U_{T}}{\partial \omega_{j}}=U_{M} \ldots U_{j+1} \frac{\partial U_{j}}{\partial \omega_{j}} U_{j-1} \ldots U_{1}, \\
\frac{\partial^{2} U_{T}}{\partial \omega_{j}^{2}}=U_{M} \ldots U_{j+1} \frac{\partial^{2} U_{j}}{\partial \omega_{j}^{2}} U_{j-1} \ldots U_{1}, \\
\frac{\partial^{2} U_{T}}{\partial \omega_{j} \partial \omega_{k}}=U_{M} \ldots U_{j+1} \frac{\partial U_{j}}{\partial \omega_{j}} U_{j-1} \ldots \\
U_{k+1} \frac{\partial U_{k}}{\partial \omega_{k}} U_{k-1} \ldots U_{1} .
\end{gathered}
$$

An analogous approach is used for the QHO model. Again we build the constant protocol Hamiltonian matrix

$$
H(\omega)=\frac{1}{2}\left[\begin{array}{cc}
\omega^{2} & 0 \\
0 & 1
\end{array}\right]
$$

and we compute the symplectic matrix

$$
S(\omega, t)=e^{J H(\omega) t}
$$

where

$$
J=\left[\begin{array}{cc}
0 & 1 \\
-1 & 0
\end{array}\right]
$$

Since the final time symplectic matrix is $S(T)=S_{M} \ldots S_{1}$ and since the Bogoliubov coefficient $\beta$, completely determining the objective value, can be ultimately related to $S(T)$ 


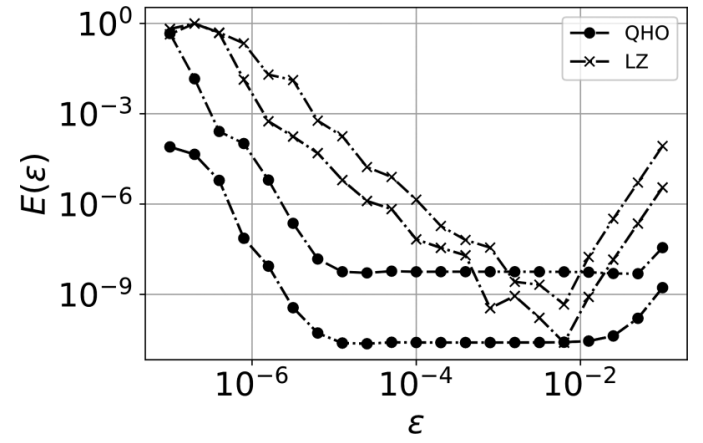

FIG. 6. Overlap error $E(\epsilon)$ between exact and approximate Hessian eigenvectors, as a function of the step in the FD computation, $\epsilon$, for the QHO model (dots) and the LZ model (crosses). Only those eigenvectors associated with nonzero eigenvalues are presented.

[21], the derivatives of the objective are obtained through the building blocks: $S(\omega), \frac{d S(\omega)}{d \omega}$, and $\frac{d^{2} S(\omega)}{d \omega}$.

\section{APPENDIX C: BENCHMARKING THE FD APPROXIMATIONS}

In Appendix A we found a general bound on the error of the FD computation of the Hessian. Let us explicitly quantify the quality of its eigenvectors individually:

$$
E_{i}=1-v_{i}^{T} \tilde{v}_{i}
$$

where $v_{i}$ and $\tilde{v}_{i}$ denote the $i$ th exact and approximate Hessian eigenvectors. Since degenerate subspaces cannot be associated with a unique choice of eigenvectors, there is no point in trying to measure an error associated with those eigenvectors in the null subspace. Moreover, the projection operation only requires those eigenvectors associated with nonzero eigenvalues, such that any component of the motion out of the null subspace can be neglected. Figure 6 presents the eigenvector overlap error $E_{i}$ as a function of $\epsilon$. Two curves, corresponding to the only two eigenvectors not belonging to the null space of $\mathbb{H}$, are plotted for each model. In both cases, excellent approximations are found, with errors below $10^{-8}$. The results agree with the estimations and the ground is set for attempting the FD navigation.

Summarizing, we first gave a rough estimation of the optimal $\epsilon$ and later refined it using the exact eigenvectors to build the error as a function of the step (Fig. 6). Unfortunately, the exact Hessian is available only in very few simple situations. To go beyond this situation, consider the following derivativefree calibration mechanism. A solution is initialized and a random direction in parameter space is chosen. A trajectory following the projection of this random direction into solution space is generated and the final infidelity is recorded. Ideally, if the step in the trajectory is sufficiently small and the eigenvectors involved in the projection operation are faithfully approximated, the infidelity along the trajectory will remain optimal. Restarting the initial solution and building new trajectories for different values of $\epsilon$, we plot the final infidelity as a function of this parameter (see Fig. 7). Two different $M=6$ solutions to the $\mathrm{QHO}$ problem were tested (dots and crosses), running 1000 iterations. The fundamental behavior

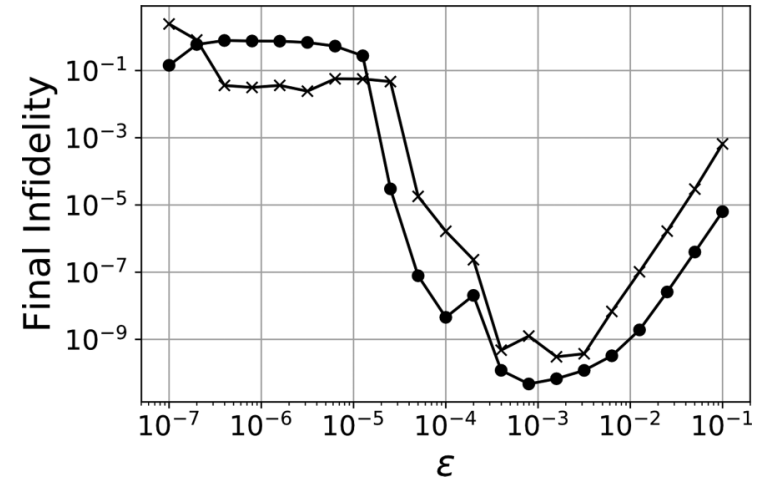

FIG. 7. Calibration procedure. A solution to the $\mathrm{QHO}$ problem with $M=6$ parameters is initialized. A direction in control space is chosen at random and a navigation sequence following its projection onto solution space is started. After 1000 iterations with a step of $h=0.1$, the final infidelity is recorded. The solution is initialized again and a navigation following the same randomly selected direction, this time with a new step in the FD scheme, is initiated. The final infidelity is plotted as a function of $\epsilon$ (black dots), allowing for the selection of optimal $\epsilon$ without having to compute the exact eigenvectors (see Fig. 6). A second random direction (black crosses) yields similar results.

in Fig. 6 is recovered, this time without any computation of the exact Hessian. The step suggested by the calibration is $\epsilon_{\mathrm{opt}}^{\mathrm{cal}} \approx 10^{-3}$.

To further validate the method, in Fig. 8 we show the evolution of the cost and the infidelity along the approximate trajectory (red circles) and compare it with the exact descent (dotted lines). Albeit greater, the infidelity in the approximate trajectory remains practically zero. Notably, the secondary cost trajectories are identical. Of

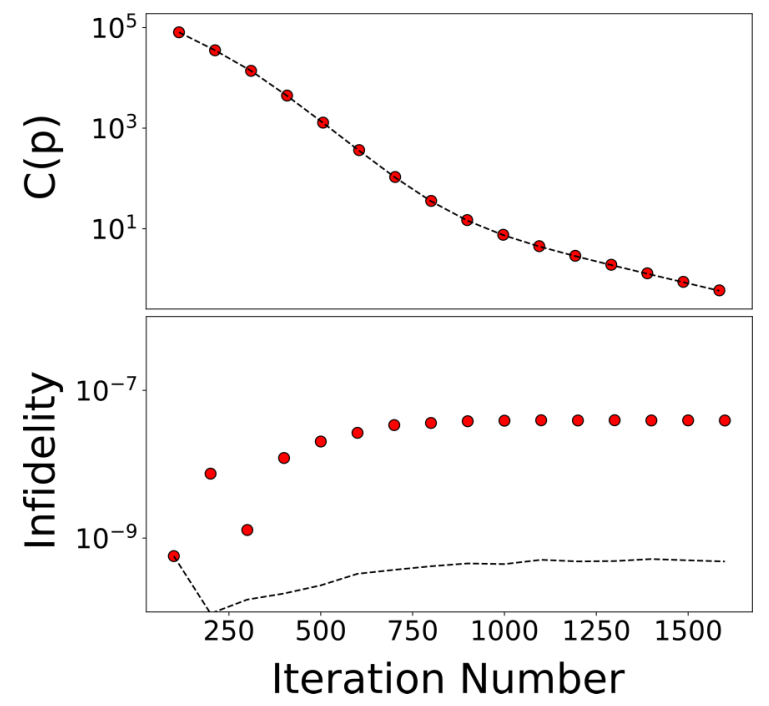

FIG. 8. Exact and approximate trajectories compared: (a) Fourier cost and (b) infidelity evolution in the exact (dotted black lines) and approximate (scattered red circles) trajectories of Fig. 4(a). Cost trajectories are indistinguishable while the infidelity remains below $10^{-7}$ in both cases. 


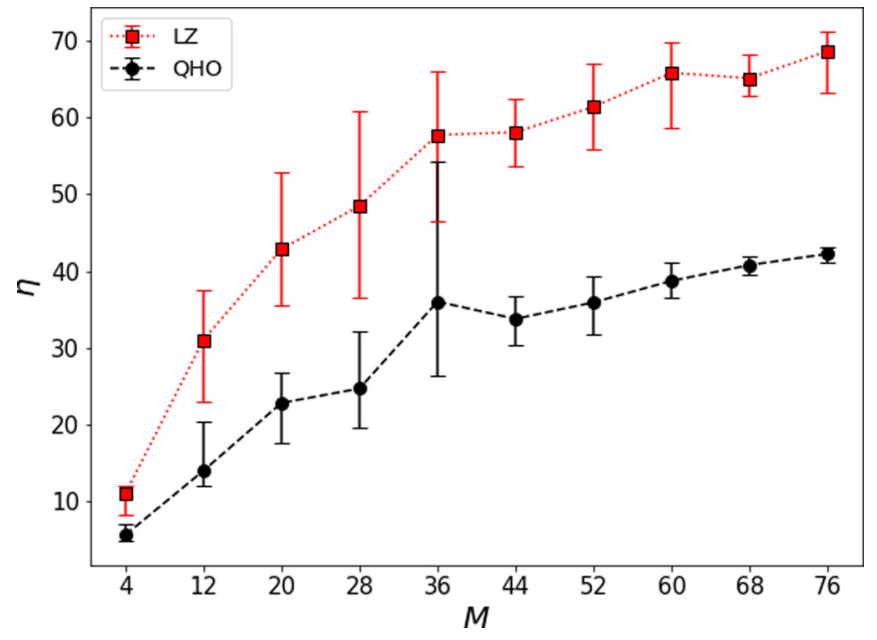

FIG. 9. Run-time analysis. Fifty random fields are initialized for different values of $M$, the Hessian eigenvectors are computed, and the run time is recorded. The mean value of $\eta$, the relative duration of the FD computations with respect to the exact ones, is plotted for both the LZ model (red squares) and the QHO model (black circles). The error bars show maximum and minimum values of $\eta$. course, there is a run-time advantage when using the exact Hessian.

Finally, we compare the run-time performance of both FD and exact schemes in Fig. 9. We initialize 50 random controls for different values of $M$ and compute the Hessian eigenvectors with both the exact and FD approaches. We define $\eta$ as the ratio between FD and exact run times. Figure 9 displays the mean value of $\eta$ as a function of $M$, for each of the models studied (the error bars show maximum and minimum values). Note that for both the LZ (in red squares) and the QHO (in black circles) the ratio has a similar tendency, increasing with $M$. This is reasonable, since the FD Hessian computation in Eq. (A1) executes $O\left(M^{2}\right)$ fidelity evaluations while the exact routines involve just a single evolution together with the evaluation of precomputed symbolic expressions for the first and second partial derivatives of the propagator. Of course, the exact computation of the Hessian cannot be employed beyond very simple situations (in our experience, the symbolic computations break for $N>3$ ) and thus approximate approaches become vital. Although not excellent in terms of speed, the FD computation is very accurate (if properly calibrated), allowing for the execution of navigation routines in systems beyond the exactly solvable.
[1] N. Gisin and R. Thew, Nat. Photonics 1, 165 (2007).

[2] I. M. Georgescu, S. Ashhab, and F. Nori, Rev. Mod. Phys. 86, 153 (2014).

[3] R. Barends, A. Shabani, L. Lamata, J. Kelly, A. Mezzacapo, U. Las Heras, R. Babbush, A. G. Fowler, B. Campbell, Y. Chen et al., Nature (London) 534, 222 (2016).

[4] J. Yin, Y. Cao, Y.-H. Li, S.-K. Liao, L. Zhang, J.-G. Ren, W.-Q. Cai, W.-Y. Liu, B. Li, H. Dai et al., Science 356, 1140 (2017).

[5] H. Bernien, S. Schwartz, A. Keesling, H. Levine, A. Omran, H. Pichler, S. Choi, A. S. Zibrov, M. Endres, M. Greiner et al., Nature (London) 551, 579 (2017).

[6] A. Acín, I. Bloch, H. Buhrman, T. Calarco, C. Eichler, J. Eisert, D. Esteve, N. Gisin, S. J. Glaser, F. Jelezko et al., New J. Phys. 20, 080201 (2018).

[7] A. P. Peirce, M. A. Dahleh, and H. Rabitz, Phys. Rev. A 37, 4950 (1988).

[8] J. Somlói, V. A. Kazakov, and D. J. Tannor, Chem. Phys. 172, 85 (1993).

[9] C. Brif, R. Chakrabarti, and H. Rabitz, New J. Phys. 12, 075008 (2010).

[10] S. J. Glaser, U. Boscain, T. Calarco, C. P. Koch, W. Köckenberger, R. Kosloff, I. Kuprov, B. Luy, S. Schirmer, T. Schulte-Herbrggen et al., European Phys. J. D 69, 279 (2015).

[11] N. Khaneja, T. Reiss, C. Kehlet, T. Schulte-Herbrüggen, and S. J. Glaser, J. Magn. Reson. 172, 296 (2005).

[12] S. Machnes, U. Sander, S. J. Glaser, P. de Fouquières, A. Gruslys, S. Schirmer, and T. Schulte-Herbrüggen, Phys. Rev. A 84, 022305 (2011).

[13] S. Machnes, E. Assémat, D. Tannor, and F. K. Wilhelm, Phys. Rev. Lett. 120, 150401 (2018).
[14] P. Rembold, N. Oshnik, M. M. Müller, S. Montangero, T. Calarco, and E. Neu, AVS Quantum Sci. 2, 024701 (2020).

[15] T. Caneva, T. Calarco, and S. Montangero, Phys. Rev. A 84, 022326 (2011).

[16] H. A. Rabitz, M. M. Hsieh, and C. M. Rosenthal, Science 303, 1998 (2004).

[17] Z. Shen, M. Hsieh, and H. Rabitz, J. Chem. Phys. 124, 204106 (2006).

[18] H. Rabitz, T.-S. Ho, M. Hsieh, R. Kosut, and M. Demiralp, Phys. Rev. A 74, 012721 (2006).

[19] V. Beltrani, J. Dominy, T.-S. Ho, and H. Rabitz, J. Chem. Phys. 134, 194106 (2011).

[20] K. W. Moore and H. Rabitz, J. Chem. Phys. 137, 134113 (2012).

[21] M. Larocca, E. Calzetta, and D. A. Wisniacki, Phys. Rev. A 101, 023410 (2020).

[22] C. Zener and R. H. Fowler, Proc. R. Soc. A 137, 696 (1932).

[23] G. C. Hegerfeldt, Phys. Rev. Lett. 111, 260501 (2013).

[24] M. Larocca, P. M. Poggi, and D. A. Wisniacki, J. Phys. A: Math. Theor. 51, 385305 (2018).

[25] P. Salamon, K. H. Hoffmann, Y. Rezek, and R. Kosloff, Phys. Chem. Chem. Phys. 11, 1027 (2009).

[26] O. Abah, J. Roßnagel, G. Jacob, S. Deffner, F. Schmidt-Kaler, K. Singer, and E. Lutz, Phys. Rev. Lett. 109, 203006 (2012).

[27] D. Stefanatos, J. Ruths, and J.-S. Li, Phys. Rev. A 82, 063422 (2010).

[28] D. Stefanatos, IEEE Trans. Autom. Control 62, 4290 (2017).

[29] R. Kosloff and Y. Rezek, Entropy 19, 136 (2017).

[30] E. A. Calzetta and B.-L. B. Hu, Nonequilibrium Quantum Field Theory (Cambridge University, Cambridge, England, 2008).

[31] B. T. Kiani, S. Lloyd, and R. Maity, arXiv:2001.11897 (2020). 
[32] D. L. Goodwin and I. Kuprov, J. Chem. Phys. 143, 084113 (2015).

[33] J. H. M. Jensen, F. S. Møller, J. J. Sørensen, and J. F. Sherson, arXiv:2005.09943 (2020).

[34] J. Nocedal and S. Wright, Numerical Optimization (Springer, New York, 2006).
[35] A. Meurer, C. P. Smith, M. Paprocki, O. Čertík, S. B. Kirpichev, M. Rocklin, A. Kumar, S. Ivanov, J. K. Moore, S. Singh, T. Rathnayake, S. Vig, B. E. Granger, R. P. Muller, F. Bonazzi, H. Gupta, S. Vats, F. Johansson, F. Pedregosa, M. J. Curry, A. R. Terrel, v. Roučka, A. Saboo, I. Fernando, S. Kulal, R. Cimrman, and A. Scopatz, Peer J. Comput. Sci. 3, e103 (2017). 